\title{
microRNA-181a promotes the proliferation of hypertrophic scar fibroblasts and inhibits their apoptosis via targeting phosphatase and tensin homolog
}

\author{
Yanpeng Zhou ${ }^{1}$, Xun Shi ${ }^{1}$, Shengfei $\mathrm{Li}^{2}$, Quan Gan ${ }^{1}$, Zhengjun Cui ${ }^{3}$ \\ ${ }^{1}$ Traumatic Surgery, Xinxiang Central Hospital, Xinxiang, China; ${ }^{2}$ The Fourth Clinical College of Xinxiang Medical University, Xinxiang, China; \\ ${ }^{3}$ Department of Burn Surgery, The First Affiliated Hospital of Zhengzhou University, Zhengzhou, China \\ Contributions: (I) Conception and design: Y Zhou; (II) Administrative support: X Shi; (III) Provision of study materials or patients: Y Zhou, Q Gan; \\ (IV) Collection and assembly of data: All authors; (V) Data analysis and interpretation: S Li, Z Cui; (VI) Manuscript writing: All authors; (VII) Final \\ approval of manuscript: All authors. \\ Correspondence to: Zhengjun Cui. Department of Burn Surgery, The First Affiliated Hospital of Zhengzhou University, Zhengzhou 450052, China. \\ Email: cuizhengjun666@126.com.
}

Background This study aimed to compare the expression of microRNA (miR)-181a and phosphatase and
tensin homolog (PTEN) in hypertrophic scar tissue and cells and to explore the effects of miR-181a and
PTEN on the proliferation and apoptosis of the human scar fibroblast cell line HSFb.
Methods: HSFb cells were transfected with miR-negative control (miR-NC), miR-181a mimics, miR-
181a inhibitor, pcDNA3.1-PTEN (pc-PTEN), or small interfence-PTEN (si-PTEN) plasmid using a
Lipofectamine 2000 transfection kit. The effects of miR-181a and PTEN on the proliferation and apoptosis
of HSFb were determined using a Cell Counting Kit (CCK)-8 experiment and flow cytometry, respectively.
The effects of miR-181a and PTEN on the expression of apoptosis-related proteins in HSFb, including type
I collagen (Col-1) and type III collagen (Col-3), were measured by western blot. Finally, the relationship
between miR-181a and PTEN was explored by the dual-luciferase reporter gene experiment.

Results: The miR-181a in hypertrophic scar tissues and HSFb were significantly up-regulated compared to embryo skin fibroblast (ESF-1) cells and normal tissues $(\mathrm{P}<0.05)$, whereas the opposite results were seen for PTEN expression $(\mathrm{P}<0.05)$. Inhibiting miR-181a or upregulating the expression of PTEN significantly suppressed the proliferation of HSFb $(\mathrm{P}<0.05)$ and induced their apoptosis $(\mathrm{P}<0.05)$. Western blot revealed that inhibiting and upregulating miR-181a and PTEN, respectively, decreased the expression of the B-cell lymphoma-2 (Bcl-2), Col-1, and Col-3 proteins in $\mathrm{HSFb}$, but significantly up-regulated the expression of Bcl-2-associated X protein (Bax), cleaved caspase-3 (c-caspase-3), and cleaved caspase-9 (c-caspase-9) $(\mathrm{P}<0.05)$. The dual-luciferase reporter gene experiment results confirmed PTEN to be the downstream target gene of miR-181a. Simultaneous upregulation of miR-181a and PTEN expression had no significant effect on the proliferation and apoptosis of HSFb.

Conclusions: miR-181a promotes the up-regulation of Col-1 and Col-3, and regulates the proliferation and apoptosis of HSFb by targeting PTEN, thereby enhancing the formation of hypertrophic scarring (HS). Therefore, miR-181a and PTEN may be potential therapeutic targets for the treatment of HS.

Keywords: Hypertrophic scar; microRNAs (miRNAs or miRs); phosphatase and tensin homolog (PTEN); proliferation; apoptosis

Submitted Jan 31, 2021. Accepted for publication Apr 17, 2021.

doi: 10.21037/apm-21-604

View this article at: http://dx.doi.org/10.21037/apm-21-604 


\section{Introduction}

The skin is the human body's largest organ. The healing process after injury to the skin is long. The ideal scar is flat and almost invisible; however, excessive fibrous tissue repair during the wound healing process can lead to hypertrophic scarring (HS) (1). Although HS is not a malignant disease, it can seriously affect an individual's daily life.

Studies have shown that microRNAs (miRNAs or miRs) may be involved in the formation and development of HS. miRNAs are a type of non-coding single-stranded small RNA with an approximate length of 21-25 nucleotides. By mediating post-transcriptional regulation, they exert various biological functions, including proliferation, apoptosis, and invasion (2). For instance, Zhang et al. reported that the downregulation of miR-137 expression can induce the proliferation and metastasis of skin fibroblasts, and promote HS formation and development (3). Furthermore, a study by $\mathrm{Wu}$ et al. showed that miR-155 was downregulated in both HS tissues and HS-derived fibroblasts, and therefore suggested it as a potential therapeutic target for HS (4). Through a miRNA microarray analysis, Rang et al. observed the significantly up-regulation of miR-181a in HS tissues and cells, and that miR-181a promoted the proliferation of fibroblasts and inhibited their apoptosis (5).

The phosphatidylinositol kinase 3/protein kinase B (PI3K/AKT) signaling pathway has been shown to be closely related to HS formation (6), and multiple reports have demonstrated that its activation is mediated by phosphatase and tensin homolog (PTEN) (7). Through the mining of the ENCORI gene bank, PTEN was found to be the downstream target gene of miR-181a. Nonetheless, few studies to date have investigated the role of miR-181a in HS. Therefore, in the hope of unveiling a new therapeutic strategy for HS, the present study aimed to verify the targeting relationship between miR-181a and PTEN, and to explore the effects of miR-181a and PTEN on the proliferation and apoptosis of HS fibroblasts at the cellular level. We present the following article in accordance with the MDAR checklist (available at http://dx.doi. org/10.21037/apm-21-604).

\section{Methods}

\section{Experimental materials and main reagents}

HS tissues and the normal skin tissues from 20 patients who underwent scar resection in our hospital between March 2016 and December 2019 were collected. Among them were 7 males and 13 females between the ages of 18 and 62 years old. All patients were diagnosed with HS, confirmed via clinical and pathological diagnosis. All procedures performed in this study involving human participants were in accordance with the Declaration of Helsinki (as revised in 2013). Each patient signed an informed consent form, and approval for the study was granted by the ethics committee of Xinxiang Central Hospital.

The human embryo skin fibroblast cell line ESF-1 and the human HS fibroblast cell line HSFb were purchased from the American Type Culture Collection. Fetal bovine serum (FBS) and Roswell Park Memorial Institute (RPMI) 1640 Medium were obtained from Gibco (USA, California), and penicillin-streptomycin solution (1\%) was purchased from Merck KGaA (Germany, Darmstadt). TRIzol reagent and a Lipofectamine 2000 transfection kit were obtained from Invitrogen (USA, California). A miScript reverse transcription kit was purchased from Qiagen GmbH (Germany, Hilden City). A SYBR premix Ex TaqTM II kit and a Cell Counting Kit (CCK)-8 kit were purchased from Tongren Institute of Chemistry (Japan, Kumamoto Prefecture). A BCA kit and an electrochemiluminescence (ECL) kit were acquired from Beijing Pulilai Gene Technology Co., Ltd. An Annexin V-FITC/PI apoptosis detection kit was purchased from BD Biosciences (USA, New Jersey). Primary antibodies for type I collagen (Col-1), type III collagen (Col-3), B-cell lymphoma-2 (Bcl-2), Bcl-2-associated $\mathrm{X}$ protein (Bax), caspase-3, cleaved caspase-3 (c-caspase-3), caspase-9, and cleaved caspase-9 (c-caspase-9), together with horseradish peroxidase-labeled goat anti-rabbit immunoglobulin G (IgG) secondary antibody, were purchased from Cell Signaling Technology (USA, Essex).

\section{Cell culture and transfection}

ESF1 cells and HSFb were seeded in a 6 -well plate at a density of $5 \times 10^{4}$ cells/well and cultured in RPMI 1640 Medium containing $10 \%$ FBS and $1 \%$ penicillinstreptomyces. The cells were then placed at $37{ }^{\circ} \mathrm{C}$ with $5 \% \mathrm{CO}_{2}$ in a constant-temperature incubator. After 24 hours of incubation, HSFb were transfected with miR-negative control (miR-NC), miR-181a mimics, miR-181a inhibitor, pcDNA3.1-PTEN (pc-PTEN), or small interfence-PTEN (si-PTEN) plasmids (sequence: 5'-GACGGGAAGACAAGUUCAUTT-3') according to the instructions accompanying the Lipofectamine 2000 
Table 1 Primer sequences

\begin{tabular}{ll}
\hline Gene & Sequence $\left(5^{\prime}-3^{\prime}\right)$ \\
\hline miR-181a & F: ACACTCCAGCTGGGAACATTCAACGCTGTCG \\
& R: GGTGTCGTGGAGTCGGCAATTCAGTTGAG \\
U6 & F: CTCGCTTCGGCAGCACA \\
& R: AACGCTTCACGAATTTGCGT \\
PTEN & F: TGGATTCGACTTAGACTTGACCT \\
& R: GGTGGGTTATGGTCTTCAAAAGG \\
GAPDH & F: CTTTGGTATCGTGGAAGGACTC \\
& R: GTAGAGGCAGGGATGATGTTCT
\end{tabular}

transfection kit. The transfected cells were then incubated at $37^{\circ} \mathrm{C}$ in a constant-temperature incubator containing $5 \%$ $\mathrm{CO}_{2}$ for a further 48 hours before subsequent experiments.

\section{Determination of miR-181a and PTEN expression}

Total RNA was extracted from normal skin (normal) tissue, HS tissue, ESF-1 cells, and HSFb using TRIzol reagent. The miScript reverse transcription (RT) kit was used for synthesis of the corresponding cDNA. The following condition was used for RT: $16^{\circ} \mathrm{C}$ for 30 minutes, $42{ }^{\circ} \mathrm{C}$ for 30 minutes, and $85^{\circ} \mathrm{C}$ for 5 minutes, then maintenance at $4^{\circ} \mathrm{C}$. The SYBR Premix Ex Taq ${ }^{\mathrm{TM}}$ II kit was then used for the measurement of miR-181a and PTEN expression. The thermal cycling conditions for polymerase chain reaction (PCR) were as follows: $95^{\circ} \mathrm{C}$ for 5 minutes, $95^{\circ} \mathrm{C}$ for 15 seconds, and $60{ }^{\circ} \mathrm{C}$ for 30 seconds, for a total of 40 cycles. The $2^{-\Delta \Delta \mathrm{Ct}}$ method was used to calculate the relative expression levels of miR-181a and PTEN with U6 and GAPDH used as an internal reference, respectively. The primer sequences used in this study are listed in Table 1 .

\section{Detection of the levels of PTEN protein and other proteins by western blot}

The lysate was used to extract total protein from normal skin tissue, HS tissue, ESF-1 cells, and HSFb. The protein concentration was then measured using the BCA kit. After that, the $10 \%$ sodium dodecyl sulphate-polyacrylamide gel electrophoresis (SDS-PAGE) was used to separate the proteins $(30 \mu \mathrm{g} / \mathrm{lane})$, which were then transferred to a polyvinylidene difluoride (PVDF) membranes. Next, the membrane was sealed with $5 \%$ skimmed milk powder for 1.5 hours at room temperature. Then, diluted antibodys of PTEN (1:1,000), Col-1 (1:1,000), Col-3 (1:1,000), Bcl2 (1:1,000), Bax $(1: 1,000)$, caspase-3 (1:1,000), c-caspase-3 $(1: 1,000)$, caspase-9 $(1: 1,000)$, and c-caspase- $9(1: 1,000)$ was added, before incubation overnight at $4{ }^{\circ} \mathrm{C}$. After 3 washes with phosphate-buffered saline (PBS) buffer, goat antirabbit IgG secondary antibody $(1: 2,000)$ was added to the membrane, which was then incubated at room temperature for 1 hour. After incubation, the ECL kit was used for blot development. The gray value of each band was analyzed with Image J software, and the relative expression level of each protein was calculated.

\section{Verification of the relationship between miR-181a and PTEN}

Wild-type PTEN (WT-PTEN) and mutant PTEN (MUTPTEN) were cloned into the pmiR-RB-ReportTM dualluciferase reporter plasmid vector. HSFb in the logarithmic growth phase were seeded in a 24-well plate at a density of $1 \times 10^{4}$ cells/well and incubated overnight at $37{ }^{\circ} \mathrm{C}$ with $5 \%$ $\mathrm{CO}_{2}$ in a constant-temperature incubator. Then, $\mathrm{HSFb}$ were transfected with miR-NC and WT-PTEN, miRNC and MUT-PTEN, miR-181a and WT-PTEN, or miR-181a and MUT-PTEN according to the instructions accompanying the Lipofectamine 2000 transfection kit. After 48 hours of transfection, the relative luciferase activity was measured in each well using Renilla luciferase activity as a reference.

\section{Measurement of HSFb proliferation by CCK-8 experiment}

$\mathrm{HSFb}$ were cultured in 96-well plates at a density of $5 \times 10^{3}$ cells/well. Either miR-NC, miR-181a mimics, miR-181a inhibitor, pc-PTEN, or si-PTEN was used to transfect the cells, and then cultured at $37{ }^{\circ} \mathrm{C}$ with $5 \% \mathrm{CO}_{2}$ in a constant temperature incubator for a further 48 hours after transfection. Next, $10 \mu \mathrm{L}$ of CCK- 8 solution and $90 \mu \mathrm{L}$ of RPMI 1640 Medium were added to each well. After incubation for 4 hours, a microplate reader was used to measure the absorbance value of each well at $450 \mathrm{~nm}$.

\section{Apoptosis of HSFb measured by flow cytometry}

$\mathrm{HSFb}$ in the logarithmic growth phase were transfected with either miR-NC, miR-181a mimics, miR-181a inhibitor, pc-PTEN, or si-PTEN, and then cultured at 

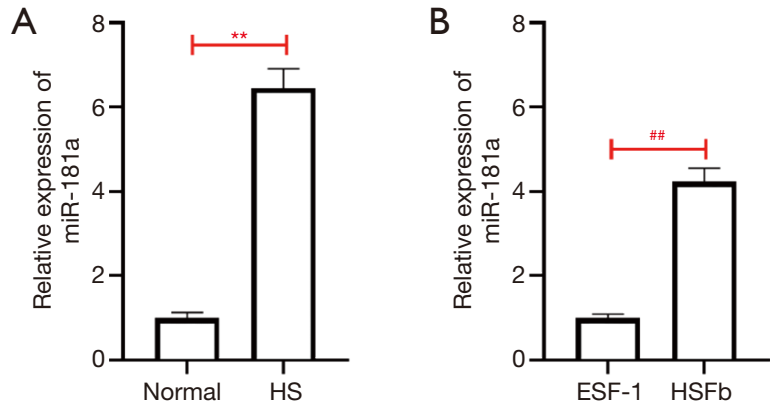

Figure 1 Relative expression levels of miR-181a in hypertrophic scar (HS) tissues and human scar fibroblasts (HSFb). (A,B) The expression level of miR-181a in HS tissues and cells measured by quantitative reverse transcription-polymerse chain reaction. ${ }^{* *} \mathrm{P}<0.01$ vs. normal group; ${ }^{\# \#} \mathrm{P}<0.01$ vs. normal group.

$37{ }^{\circ} \mathrm{C}$ with $5 \% \mathrm{CO}_{2}$ in a constant-temperature incubator. After 48 hours of incubation, the cells were washed 3 times with pre-chilled PBS buffer and then stained in the dark by using the Annexin V-FITC/PI Apoptosis Detection Kit. After 15 minutes of staining, cell apoptosis was then detected by flow cytometry.

\section{Statistical analysis}

SPSS 20.0 software developed by IBM (Armonk, New York, USA) was used for the experimental data analysis, and the GraphPad Prism 8.2.1 software was used to draw statistical graph. All tests were repeated 3 times, and the measurement data values were expressed as mean \pm standard deviation $(\bar{x} \pm s)$. Data between the 2 groups that conformed to a normal distribution were analyzed using $t$-test. Data between multiple groups were analyzed by one-way analysis of variance (ANOVA) and randomized block design ANOVA. $\mathrm{P}<0.05$ was used for statistically significance.

\section{Results}

\section{Expression of miR-181a in HS tissues and cells}

Twenty HS tissues (HS group) and corresponding normal skin tissues (normal group) were used in this study. The expression of miR-181a in the HS group was significantly higher than the normal group $(\mathrm{P}<0.05)$. Furthermore, compared with ESF-1 cells, HSFb exhibited a significantly elevated expression of miR-181a $(\mathrm{P}<0.05)$, as shown in Figure 1.

\section{PTEN expression in HS tissues and cells}

As shown in Figure $2 A, B$, the RT-qPCR and WB results revealed a low expression of PTEN messenger RNA (mRNA) in HS tissues and HSFb $(\mathrm{P}<0.05)$. ENCORI prediction showed PTEN to be the downstream target gene of miR-181a; the targeted binding sequence is shown in Figure 2C. After transfection with miR-181a mimics and miR-181a inhibitor, the expression levels of miR181a in HSFb were significantly increased and decreased, respectively $(\mathrm{P}<0.05)$, as shown in Figure $2 D$. The western blot showed that in HSFb, the expression of PTEN protein was significantly reduced following transfection with miR181a mimics, while the expression of PTEN protein was significantly increased after transfection with miR-181a inhibitor. These results indicated that miR-181a negatively regulates PTEN protein expression (Figure 2E). The dual-luciferase reporter gene experiment showed that the luciferase activity of WT-PTEN was significantly inhibited $(\mathrm{P}<0.05)$ after miR-181a transfection, although we failed to observe a similar significant effect on the luciferase activity of MUT-PTEN (Figure 2F).

\section{miR-181a regulates $H S F b$ proliferation and apoptosis in vitro}

The proliferation of $\mathrm{HSFb}$ was determined by the CCK8 experiment. As shown in Figure 3A, HSFb proliferation was significantly enhanced by miR-181a mimic transfection $(\mathrm{P}<0.05)$, whereas the transfection of miR-181a inhibitor had the opposite effect $(\mathrm{P}<0.05)$. The apoptosis of $\mathrm{HSFb}$ was detected by flow cytometry, which showed that HSFb apoptosis was significantly inhibited following transfection with miR-181a mimics $(\mathrm{P}<0.05)$, while miR-181a inhibitor transfection significantly enhanced the early-stage apoptosis of HSFb $(\mathrm{P}<0.05)$ and also significantly elevated the total apoptotic rate $(\mathrm{P}<0.05)$ (Figure $3 B)$. The levels of apoptosisrelated proteins and collagens in $\mathrm{HSFb}$ were determined by western blot. The results showed that Bcl-2 Col-1, and Col-3 protein were significantly increased after transfection with miR-181a mimics $(\mathrm{P}<0.05)$, while the levels of Bax, c-caspase- 3 , and c-caspase-9 protein were significantly inhibited $(\mathrm{P}<0.05)$. As expected, following transfection of $\mathrm{HSFb}$ with miR-181a inhibitor, the expression of Bcl2 protein was significantly reduced $(\mathrm{P}<0.05)$, as was the protein expression of Col-1 and Col-3 $(\mathrm{P}<0.05)$, whereas the expression levels of Bax, c-caspase-3, and c-caspase-9 were significantly upregulated $(\mathrm{P}<0.05)($ Figure $3 C, D)$. 

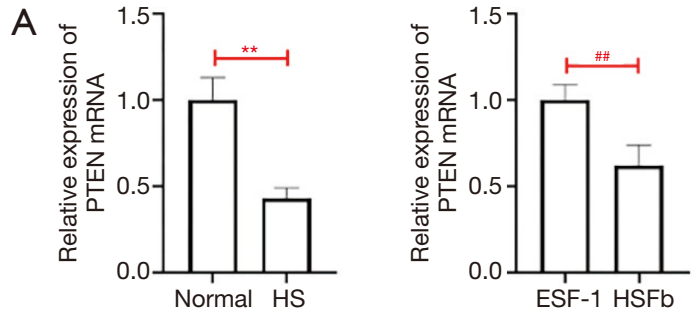

C

PTEN-WT: 5 `-uauuauuuuuccuuugGAAUGUg-3`

| || | | |

miR-181a: 3'-ugaguggcugucgcaaCUUACAa-5 '

PTEN-MUT: 5 `-uauuauuuuuccuuugCUUACAg-3`

E

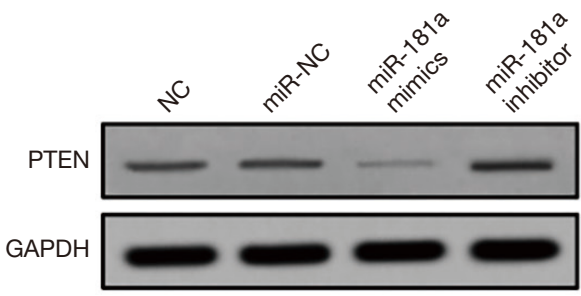

B

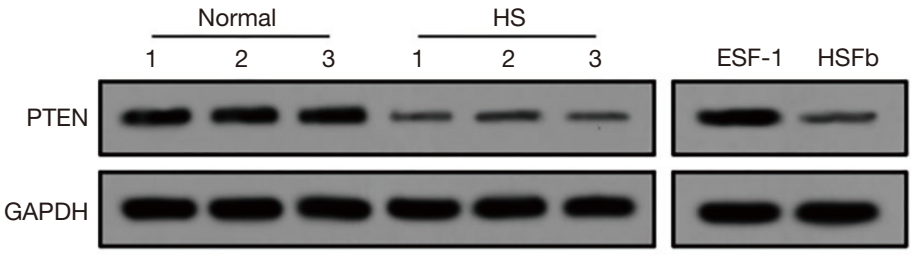

D

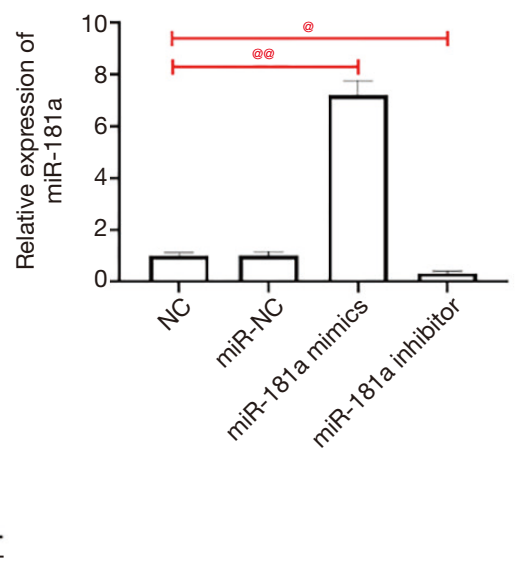

Figure 2 Relative expression levels of phosphatase and tensin homolog (PTEN) protein and mRNA in hypertrophic scar (HS) tissues and human scar fibroblasts (HSFb). (A) The expression of PTEN messenger RNA (mRNA) in HS tissues and HSFb measured by semiquantitative reverse transcription-polymerase chain reaction (RT-qPCR); (B) The expression of PTEN protein in HS tissues and HSFb measured by western blotting; (C) the target binding site of miR-181a in the PTEN gene; (D) the expression of miR-181a in HSFb measured by RT-qPCR; (E) the expression of PTEN protein in HSFb determined by western blot; (F) dual-luciferase reporter gene assay verified the targeting relationship between miR-181a and $\mathrm{PTEN}$. ${ }^{* *} \mathrm{P}<0.01$ vs. normal group; ${ }^{* \#} \mathrm{P}<0.01$ vs. embryo skin fibroblast cells (ESF-1) group; ${ }^{\circledR} \mathrm{P}<0.05$ and ${ }^{\circledR}{ }^{\circledR} \mathrm{P}<0.01$ vs. negative control $(\mathrm{NC})$ group; ${ }^{\circledR} \mathrm{P}<0.05$ vs. miR-NC group.

\section{PTEN regulates $\mathrm{HSFb}$ proliferation and apoptosis in vitro as a downstream target gene of miR-181a}

After transfection with pc-PTEN and si-PTEN plasmids, the expression of PTEN protein in $\mathrm{HSFb}$ was significantly affected $(\mathrm{P}<0.05)$ (Figure $4 A)$. The results of CCK8 experiments showed that $\mathrm{HSFb}$ proliferation was significantly inhibited following transfection with pcPTEN $(\mathrm{P}<0.05)$, whereas si-PTEN transfection had the opposite effect $(\mathrm{P}<0.05)$. Also, as shown in Figure $4 B$, the simultaneous transfection of miR-181a mimics and pcPTEN had no obvious effect on the proliferation of HSFb. The results of flow cytometry showed that the apoptotic rate of $\mathrm{HSFb}$ was significantly increased after pc-PTEN transfection $(\mathrm{P}<0.05)$, and the increase was most significant in the early apoptotic rate $(\mathrm{P}<0.05)$; in contrast, the transfection of si-PTEN inhibited the apoptosis of $\mathrm{HSFb}$ $(\mathrm{P}<0.05)$. However, as shown in Figure $4 C$, the simultaneous transfection of miR-181a mimics and pc-PTEN had no significant effect on the apoptosis rate of $\mathrm{HSFb}(\mathrm{P}<0.05)$. The western blot results showed that transfection with pcPTEN inhibited the expression of $\mathrm{Bcl}-2$ protein $(\mathrm{P}<0.05)$, as well as the expression of Col- 1 and Col-3 protein $(\mathrm{P}<0.05)$, in $\mathrm{HSFb}$, but upregulated the protein expression of Bax, c-caspase-3, and c-caspase-9 $(\mathrm{P}<0.05)$. Moreover, after siPTEN transfection, the protein expression levels of Bcl2, Col-1, and Col-3 were significantly increased in $\mathrm{HSFb}$ $(\mathrm{P}<0.05)$, whereas those of Bax, c-caspase-3, and c-caspase-9 were inhibited $(\mathrm{P}<0.05)$. Finally, simultaneous transfection of miR-181a mimics and pc-PTEN had no obvious effects on the protein expression levels of Bcl-2, c-caspase-3, c-caspase-9, Col-1, or Col-3 in HSFb (Figure 4D,E).

\section{Discussion}

HS is 1 of the most common skin conditions, occurring in 


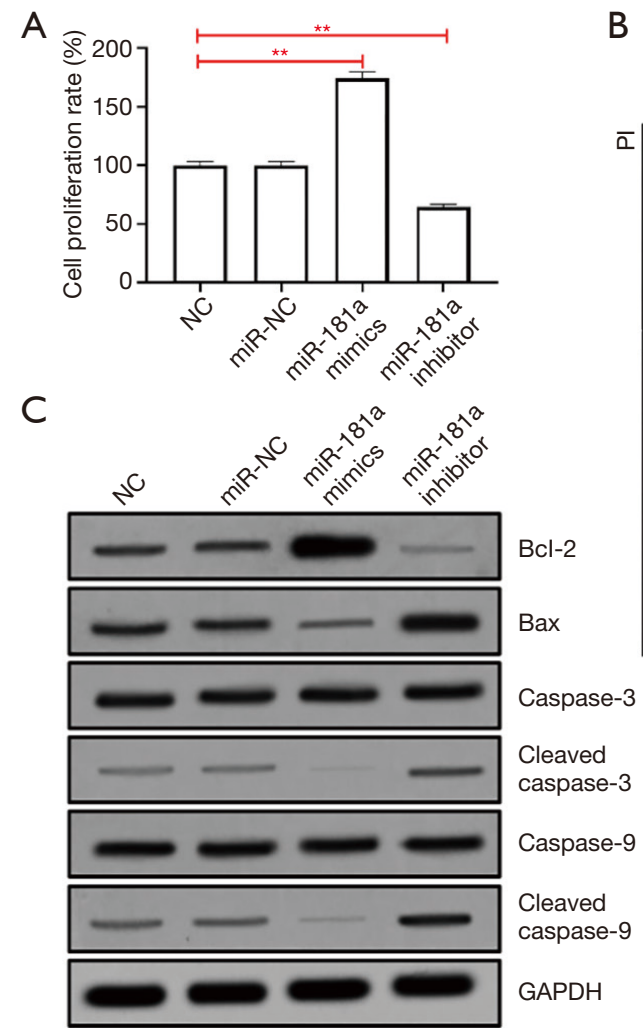

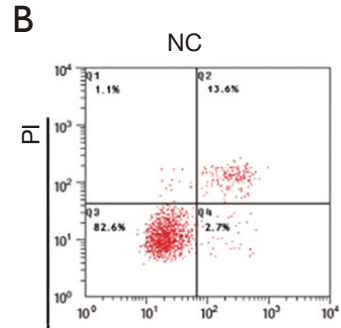

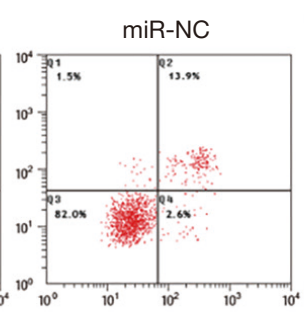

miR-181a mimics

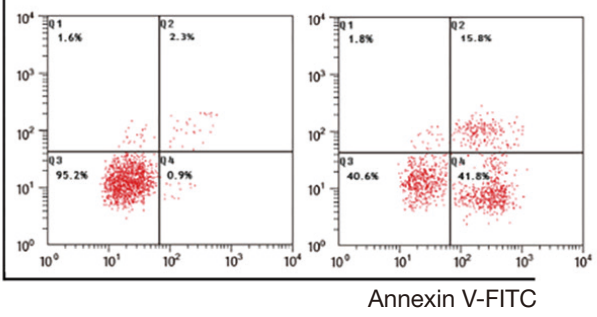

miR-181a inhibitor

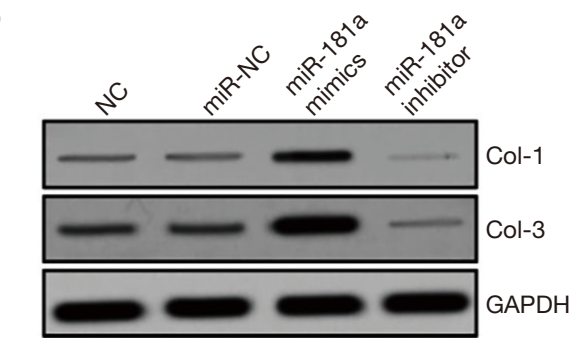

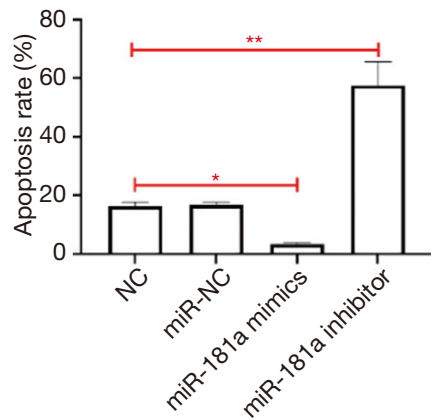

Figure 3 miR-181a regulates human scar fibroblast (HSFb) proliferation and apoptosis in vitro. (A) The effect of miR-181a on HSFb proliferation, as determined by Cell Counting Kit (CCK)-8 experiment; (B) the effect of miR-181a on HSFb apoptosis, as determined by flow cytometry; (C,D) the effect of miR-181a on the expression of apoptosis-related proteins and collagens in HSFb, measured by western blot. ${ }^{*} \mathrm{P}<0.05$ and ${ }^{* *} \mathrm{P}<0.01$ vs. negative control (NC) group.

$30 \%$ of patients who suffer burns and scalds (8). Although HS can be treated with surgery, it may recur after surgical treatment. Multiple studies have shown that fibroblasts play a role in the processes of tissue repair and wound healing. In particular, the proliferation and apoptosis of $\mathrm{HSFb}$ are critical in the formation of HS (9). Therefore, elucidating the molecular mechanisms of HSFb proliferation and apoptosis may aid in the development of new treatment methods for HS.

In recent years, numerous studies have shown that miRNAs play an important role in the pathogenesis of HS (5,10-12). Although miR-181a has been confirmed to be involved in the occurrence and development of colon cancer (13), lung cancer (14), breast cancer (15), and thyroid cancer (16), few studies have focused on its expression and biological functions in HS. Zhang et al. suggested that HS results from $\mathrm{HSFb}$ proliferation and inhibition of $\mathrm{HSFb}$ apoptosis (17). In the current study, the expression of miR181a in HS tissues was found to be significantly higher than that in normal skin tissues, and the expression of miR181a in HSFb was also significantly higher than that in ESF-1 cells. Therefore, we speculate that miR-181a may be a regulator involved in the proliferation and apoptosis of HSFb. To clarify the specific role of miR-181a in HSFb proliferation and apoptosis, we achieved the upregulation or inhibition of miR-181a expression in HSFb via transfection. The results showed that inhibiting the expression of miR181a not only significantly suppressed the proliferation of $\mathrm{HSFb}$ but also induced early apoptosis.

PTEN is a dual phosphatase located on chromosome 10q23.3 that can inhibit tumor development. It can exert an anti-apoptotic effect by regulating the activity of the PI3K/AKT signaling pathway, as well as promote cell proliferation (18). Studies have shown that the loss of PTEN expression is associated with the formation of HS $(7,19,20)$; consistent with this, our study found that the expression of PTEN mRNA in HS tissues and HSFb was significantly downregulated. miRNAs usually exert 


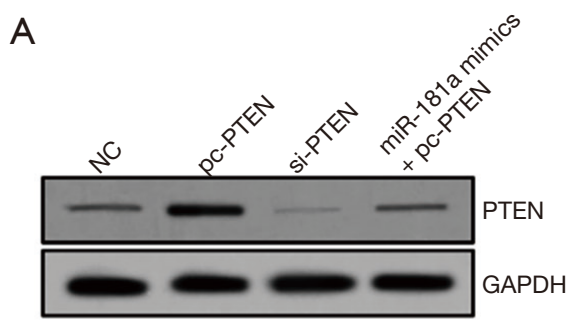

C
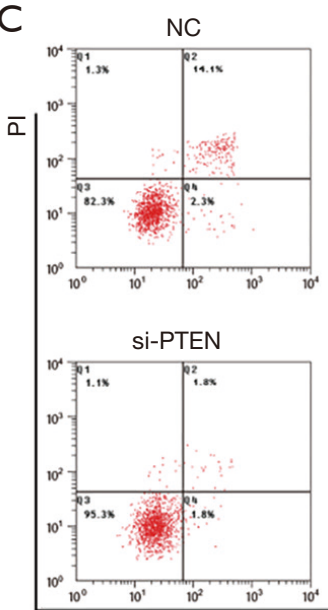

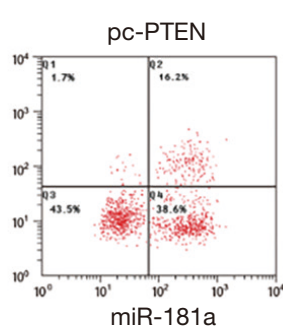

mimics + pc-PTEN

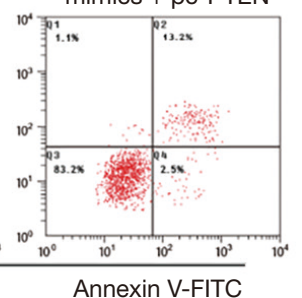

B
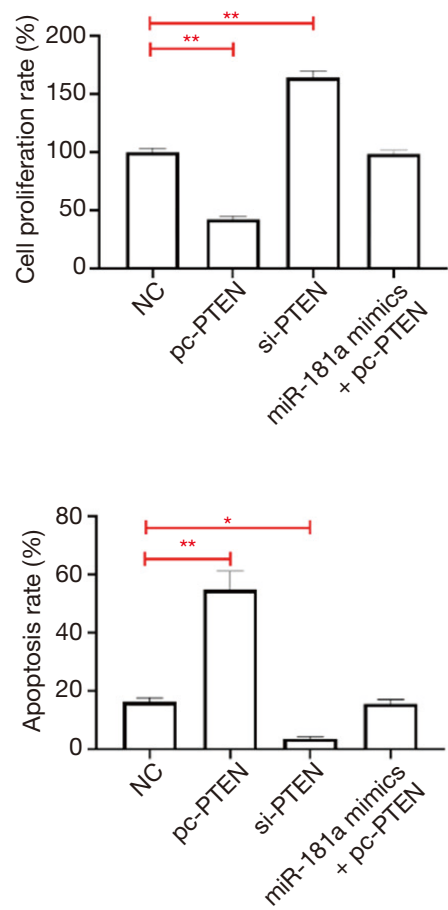

D

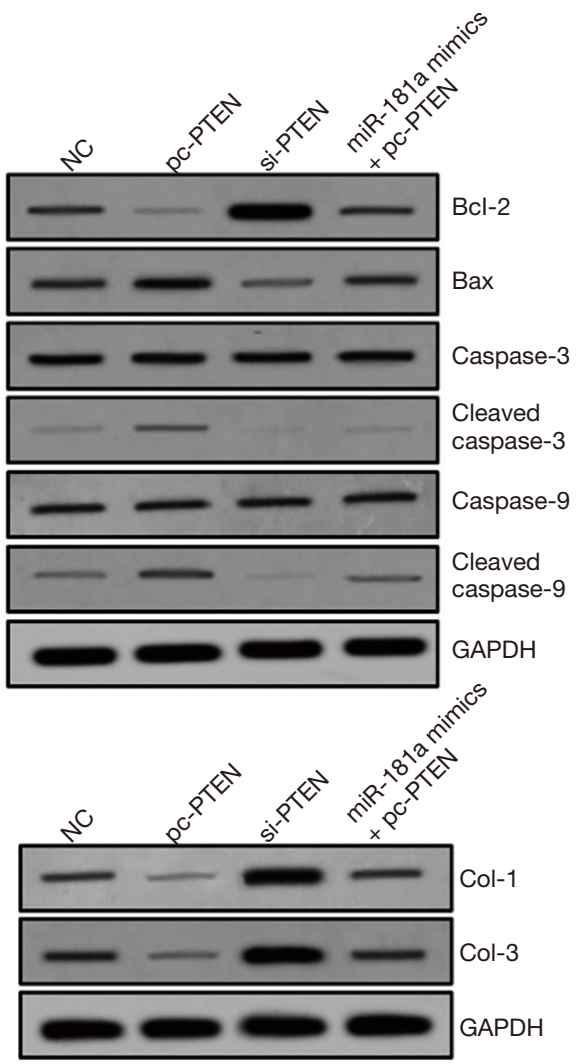

Figure 4 Phosphatase and tensin homolog (PTEN) regulates human scar fibroblast (HSFb) proliferation and apoptosis in vitro as a downstream target gene of miR-181a. (A) The expression level of phosphatase and tensin homolog (PTEN) protein in HSFb determined by western blot; (B) the effect of PTEN on HSFb proliferation determined by Cell Counting Kit (CCK)-8 experiment; (C) the effect of PTEN on HSFb apoptosis detected by the flow cytometry; $(\mathrm{D}, \mathrm{E})$ the effect of PTEN on the expression of apoptosis-related proteins and collagens in $\mathrm{HSFb}$ detected by western blot. ${ }^{*} \mathrm{P}<0.05$ and ${ }^{* *} \mathrm{P}<0.01$ vs. negative control (NC) group.

their biological functions by influencing the translation of downstream target genes. This study found that miR-181a has partially complementary sequences with PTEN at its 3 prime untranslated region. Subsequently, we performed a dual-luciferase reporter gene experiment, which also confirmed the targeting relationship between miR-181a and PTEN. Furthermore, miR-181a can negatively regulate the expression of PTEN protein. In this study, it was confirmed that the upregulation of PTEN expression in HSFb can inhibit their proliferation and induce apoptosis.

The overexpression of Col-1 and Col-3 is also considered to be 1 of the causes of HS (21). It has been demonstrated that the expression levels of Col-1 and Col-3 in HS tissues and $\mathrm{HSFb}$ are significantly increased compared to those in normal skin tissues and cells (22). The results of the present research showed that while inhibiting
miR-181a or upregulating PTEN in HSFb can significantly inhibit the expression of Col-1 and Col-3, simultaneous upregulation of miR-181a and PTEN has no obvious effect on the expression of Col-1 and Col-3 in HSFb. The above results indicate that miR-181a regulates Col-1 and Col-3 expression in HSFb by targeting PTEN; in other words, it acts as an anti-fibrotic factor in HS.

In summary, the results of this study showed that miR-181a promotes the expression of Col-1 and Col3 , and regulates the proliferation and apoptosis of $\mathrm{HSFb}$ by targeting PTEN, thus promoting the formation of HS. Therefore, miR-181a and PTEN could be potential therapeutic targets for HS. Although drugs that inhibit miR-181a have yet to be developed, drugs that upregulate PTEN, such as sorafenib, have been used clinically. Mutations or deletions of the PTEN gene are found in most 
patients with liver cancer (23), and sorafenib can upregulate the expression of PTEN through a variety of mechanisms to inhibit the occurrence of liver cancer (24). Given the regulatory effect of sorafenib on PTEN in liver cancer cells, we hypothesize that sorafenib may also upregulate the expression of PTEN in $\mathrm{HSFb}$, thereby inhibiting the formation of HS. The above hypothesis will be the focus of our next research, in which we will evaluate the effects and safety of sorafenib in the treatment of HS by performing animal experiments, and explore new ways and methods for treating HS.

\section{Acknowledgments}

Funding: None.

\section{Footnote}

Reporting Checklist: The authors have completed the MDAR checklist. Available at http://dx.doi.org/10.21037/apm-21604

Data Sharing Statement: Available at http://dx.doi. org/10.21037/apm-21-604

Conflicts of Interest: All authors have completed the ICMJE uniform disclosure form (available at http://dx.doi. org/10.21037/apm-21-604). The authors have no conflicts of interest to declare.

Ethical Statement: The authors are accountable for all aspects of the work in ensuring that questions related to the accuracy or integrity of any part of the work are appropriately investigated and resolved. All procedures performed in this study involving human participants were in accordance with the Declaration of Helsinki (as revised in 2013). This study was approved by the Xinxiang Central Hospital (No. ZXYY-KY-0398), and written informed consent was obtained from the patients and their families.

Open Access Statement: This is an Open Access article distributed in accordance with the Creative Commons Attribution-NonCommercial-NoDerivs 4.0 International License (CC BY-NC-ND 4.0), which permits the noncommercial replication and distribution of the article with the strict proviso that no changes or edits are made and the original work is properly cited (including links to both the formal publication through the relevant DOI and the license).
See: https://creativecommons.org/licenses/by-nc-nd/4.0/.

\section{References}

1. Lee H J, Jang YJ. Recent Understandings of Biology, Prophylaxis and Treatment Strategies for Hypertrophic Scars and Keloids. Int J Mol Sci 2018;19:711.

2. Chen L, Heikkinen L, Wang C, et al. Trends in the development of miRNA bioinformatics tools. Brief Bioinform 2019;20:1836-52.

3. Zhang Q, Guo B, Hui Q, et al. miR-137 Inhibits Proliferation and Metastasis of Hypertrophic Scar Fibroblasts via Targeting Pleiotrophin. Cell Physiol Biochem 2018;49:985-95.

4. Wu X, Li J, Yang X, et al. miR-155 inhibits the formation of hypertrophic scar fibroblasts by targeting HIF- $1 \alpha$ via PI3K/AKT pathway. J Mol Histol 2018;49:377-87.

5. Rang Z, Wang ZY, Pang QY, et al. MiR-181a Targets PHLPP2 to Augment AKT Signaling and Regulate Proliferation and Apoptosis in Human Keloid Fibroblasts. Cell Physiol Biochem 2016;40:796-806.

6. Zhi Y, Wang H, Huang B, et al. Panax Notoginseng Saponins suppresses TRPM7 via the PI3K/AKT pathway to inhibit hypertrophic scar formation in vitro. Burns 2020;4179:30550-7.

7. He T, Zhang Y, Liu Y, et al. MicroRNA-494 targets PTEN and suppresses PI3K/AKT pathway to alleviate hypertrophic scar formation. J Mol Histol 2019;50:315-23.

8. Tyack Z, Simons M, Spinks A, et al. A systematic review of the quality of burn scar rating scales for clinical and research use. Burns 2012;38:6-18.

9. Wang X, Chu J, Wen CJ, et al. Functional characterization of TRAP1-like protein involved in modulating fibrotic processes mediated by TGF- $\beta / \mathrm{Smad}$ signaling in hypertrophic scar fibroblasts. Exp Cell Res 2015;332:202-11.

10. Guo B, Hui Q, Xu Z, et al. miR-495 inhibits the growth of fibroblasts in hypertrophic scars. Aging (Albany NY) 2019;11:2898-910.

11. Wang X, Zhang Y, Jiang BH, et al. Study on the role of Hsa-miR-31-5p in hypertrophic scar formation and the mechanism. Exp Cell Res 2017;361:201-9.

12. Li L, Han W, Chen Y, et al. MiR-3613-3p inhibits hypertrophic scar formation by down-regulating arginine and glutamate-rich 1. Mol Cell Biochem 2021;476:1025-36.

13. Wei Z, Cui L, Mei Z, et al. miR-181a mediates metabolic shift in colon cancer cells via the PTEN/AKT pathway. 
FEBS Lett 2014;588:1773-9.

14. Ping W, Gao Y, Fan X, et al. MiR-181a contributes gefitinib resistance in non-small cell lung cancer cells by targeting GAS7. Biochem Biophys Res Commun 2018;495:2482-9.

15. Gu M, Wang L, Yang C, et al. Micro-RNA-181a suppresses progestin-promoted breast cancer cell growth. Maturitas 2018;114:60-6.

16. Le F, Luo P, Yang QO, et al. MiR-181a promotes growth of thyroid cancer cells by targeting tumor suppressor RB1. Eur Rev Med Pharmacol Sci 2017;21:5638-47.

17. Zhang J, Liu Z, Cao W, et al. Amentoflavone inhibits angiogenesis of endothelial cells and stimulates apoptosis in hypertrophic scar fibroblasts. Burns 2014;40:922-9.

18. Chen CY, Chen J, He L, et al. PTEN: Tumor Suppressor and Metabolic Regulator. Front Endocrinol (Lausanne) 2018;9:338.

19. Zhu HY, Li C, Bai WD, et al. MicroRNA-21 regulates hTERT via PTEN in hypertrophic scar fibroblasts. PLoS
One 2014;9:e97114.

20. Guo L, Chen L, Bi S, et al. PTEN inhibits proliferation and functions of hypertrophic scar fibroblasts. Mol Cell Biochem 2012;361:161-8.

21. Shi J, Xiao H, Li J, et al. Wild-type p53-modulated autophagy and autophagic fibroblast apoptosis inhibit hypertrophic scar formation. Lab Invest 2018;98:1423-37.

22. Zhou R, Zhang Q, Zhang Y, et al. Aberrant miR-21 and miR-200b expression and its pro-fibrotic potential in hypertrophic scars. Exp Cell Res 2015;339:360-6.

23. Zhou J, Li X. Association of PTEN expression with liver function and inflammatory changes in patients with liver cancer after chemotherapy. Oncol Lett 2018;16:6633-7.

24. Ruan Z P, Xu R, Lv Y, et al. PTEN enhances the sensitivity of human hepatocellular carcinoma cells to sorafenib. Oncol Res 2012;20:113-21.

(English Language Editor: J. Reynolds)

Cite this article as: Zhou Y, Shi X, Li S, Gan Q, Cui Z. microRNA-181a promotes the proliferation of hypertrophic scar fibroblasts and inhibits their apoptosis via targeting phosphatase and tensin homolog. Ann Palliat Med 2021;10(4):45634571. doi: 10.21037/apm-21-604 